\title{
ON THE DECOMPOSITION OF INVARIANT SUBSPACES
}

\author{
BY T. CRIMMINS AND P. ROSENTHAL ${ }^{1}$
}

Communicated by P. R. Halmos, July 15, 1966

1. Introduction. The main result of this paper is a decomposition theorem for invariant subspaces of operators on Banach spaces. The theorem is closely related to a decomposition theorem of F. Riesz.

We apply the theorem to the study of invariant subspaces of direct sums of operators, producing some new examples of lattices of invariant subspaces of operators on Hilbert space.

2. The Main Theorem. Let $B$ be a (complex) Banach space and $T$ a bounded linear operator on $B$. We use $\sigma(T)$ to denote the spectrum of $T$. Suppose that $\sigma(T)=\sigma_{1} \cup \sigma_{2}$, where $\sigma_{1}$ and $\sigma_{2}$ are disjoint nonempty closed sets. Then, as Riesz has shown, [4, §148], one can choose contours $\gamma_{j}, j=1,2$, with $\gamma_{j}$ surrounding $\sigma_{j}$, such that if

$$
P_{j}=-\frac{1}{2 \pi i} \int_{\gamma_{j}}(T-z)^{-1} d z
$$

then $P_{j}$ is a projection on to an invariant subspace $B_{j}$ of $T$. Moreover $B=B_{1} \bigvee B_{2}, B_{1} \cap B_{2}=\{0\}$, and $\sigma\left(T \mid B_{j}\right)=\sigma_{j}$.

We strengthen the hypothesis in Riesz's Theorem and get a stronger conclusion. If $S$ is a compact subset of the complex plane let $\eta(S)$ denote the union of $S$ and all "holes" in $S$; i.e., $\eta(S)$ is the union of $S$ and all bounded components of the complement of $S$.

THEOREM 1. If $\eta\left(\sigma_{1}\right) \cap \eta\left(\sigma_{2}\right)=\varnothing$, then every invariant subspace $M$ of $T$ has a unique decomposition of the form $M=M_{1} \bigvee M_{2}$, where $M_{j}$ is invariant under $T$ and $M_{j} \subset B_{j}, j=1,2$.

To prove the theorem we require several lemmas.

LEMmA 1. If $\eta\left(\sigma_{1}\right) \cap \eta\left(\sigma_{2}\right)=\varnothing$, then $\eta(\sigma(T))=\eta\left(\sigma_{1}\right) \cup \eta\left(\sigma_{2}\right)$.

Lemma 1 follows immediately from a property of the extended complex plane (or 2-sphere) closely related to unicoherence. (For a proof of the relevant property see $[6$, p. 60].)

Now let $M$ be any nontrivial invariant subspace for $T$ and let $R$ be the restriction of $T$ to $M$.

${ }_{1}^{1}$ The problems considered in this paper originated in the course of work done by the second author under the guidance of Professor P. R. Halmos. The authors are grateful to Professor Halmos for his suggestions regarding the preparation of the manuscript. 
Lemma 2. $\sigma(R) \subset \eta(\sigma(T))$.

In fact, more is true: if one point of a hole is in $\sigma(R)$ then the entire hole is in $\sigma(R)$.

Lemma 2 was proved by Bram [1] in the case that $T$ is a normal operator on Hilbert space. S. Parrott has observed (unpublished) that the general result follows directly from the easily established relations

$$
\delta(\sigma(R)) \subset \pi(R) \subset \pi(T) \subset \sigma(T),
$$

where $\pi$ denotes approximate point spectrum [3, p. 51], and $\delta(\sigma(R))$ denotes the boundary of $\sigma(R)$.

Lemma 3. If $f$ is a function analytic on an open set containing $\eta(\sigma(T))$, then $f(R)=f(T) \mid M$.

First of all, by Lemma 2, $f(R)$ is defined. By Riesz's functional calculus, $[4, \S 151], f(T)$ has a representation

$$
f(T)=-\frac{1}{2 \pi i} \int_{\gamma} f(z)(T-z)^{-1} d z,
$$

where $\gamma$ is a contour surrounding $\eta(\sigma(T))$. Lemma 3 now follows from the fact that if $z \in \gamma$ then $z \notin \sigma(R)$ and $z \notin \sigma(T)$, and thus $(R-z)^{-1}=(T-z)^{-1} \mid M$.

We can now prove Theorem 1. By Lemmas 1 and 2, $\sigma(R)=\tau_{1} \cup \tau_{2}$, $\tau_{j} \subset \eta\left(\sigma_{j}\right)$, (where either of the $\tau_{j}$ may be empty). Now if one considers the Riesz projections for $T$ discussed above, where the contours $\gamma_{j}$ are chosen so that $\gamma_{j}$ encloses $\eta\left(\sigma_{j}\right)$ and is disjoint from $\eta(\sigma(T))$, Lemma 3 implies that the Riesz projections for $R$, (corresponding to $\tau_{1}$ and $\tau_{2}$ ), are restrictions of the Riesz projections for $T$. The theorem follows.

REMARK. In Theorem 1 it does not suffice to assume merely that $\sigma_{1} \cap \sigma_{2}=\varnothing$. A counterexample is provided by $T=U \oplus 0$ on $H \oplus H$, where $H$ is a separable infinite-dimensional Hilbert space and $U$ is a bilateral shift on $H$. Here $\sigma_{1}=\sigma(U)=\{z:|z|=1\}$ and $\sigma_{2}=\{0\}$.

3. Application to lattices of invariant subspaces.

Definition. An abstract lattice $L$ is an $S$-lattice ( $S$ for subspace) if $L$ is order-isomorphic to the lattice of invariant subspaces of some bounded linear operator on a separable complex Hilbert space.

Theorem 2. If $\left\{L_{n}\right\}$ is a finite or countably infinite family of $S$ lattices then the direct product $\mathrm{X}_{n} L_{n}$ is an S-lattice.

To prove Theorem 2, suppose that $L_{n}$ is attained by an operator 
$T_{n}$ on the Hilbert space $H_{n}$. Let $\left\{D_{n}\right\}$ be a sequence of disjoint closed discs in the complex plane which have radii tending to zero and centers tending to zero along the real axis. By multiplying and translating the $T_{n}$ 's by appropriate scalars we can assume that they are uniformly bounded in norm and that $\sigma\left(T_{n}\right) \subset D_{n}$ for each $n$.

Now let $T=\sum_{n} \oplus T_{n}$ operate on $\sum_{n} \oplus H_{n}$. For arbitrary $m$ it is clear that

$$
\eta\left(\sigma\left(T_{m}\right)\right) \cap \eta\left(\sigma\left(\sum_{n \neq m} \oplus T_{n}\right)\right)=\varnothing .
$$

Also, because of the arrangement of the spectra of the $T_{n}$ 's it can be shown that for each $m$ we have

$$
\eta(\sigma(T))=\eta\left(\sigma\left(T_{m}\right)\right) \cup \eta\left(\sigma\left(\sum_{n \neq m} \oplus T_{n}\right)\right) .
$$

Repeated use of Theorem 1 then gives the result that if $M$ is an invariant subspace of $T$, then $M=\sum_{n} \oplus M_{n}$, where $M_{n}$ is invariant under $T_{n}$. Hence the lattice of invariant subspaces for $T$ is isomorphic to $\mathrm{X}_{n} L_{n}$.

Theorem 2 allows us to construct new examples of S-lattices. For example, if we let $L_{n}$ be a lattice known to be attainable on a finitedimensional space, for $n=1,2,3, \cdots$, then $X_{n} L_{n}$ is attainable on an infinite-dimensional space. Also the fact that the closed unit interval is an S-lattice, (attained by the Volterra integral operator-see [2] or [5]), implies that the closed unit square (with the product order) is an S-lattice.

An example of particular interest is $(\omega+1) \times(\omega+1)$. Donoghue [2] has shown that $\omega+1$ is attainable by a compact operator, and thus our construction in Theorem 2 furnishes a polynomially compact operator with lattice $(\omega+1) \times(\omega+1)$. The second author has shown, however, that $(\omega+1) \times(\omega+1)$ is not attainable by a compact operator.

\section{REFERENCES}

1. J. Bram, Subnormal operators, Duke Math. J. 22 (1955), 75-94.

2. W. F. Donoghue, The lattice of invariant subspaces of a completely continuous quasi-nilpotent transformation, Pacific J. Math. 7 (1957), 1031-1035.

3. P. R. Halmos, Introduction to Hilbert spaces, 2nd ed., Chelsea, New York, 1957.

4. F. Riesz and B. Sz-Nagy, Functional analysis, Ungar, New York, 1955.

5. D. Sarason, $A$ remark on the Volterra operator, J. Math. Anal. Appl, 12 (1965), 244-246

6. R. L. Wilder, Topology of manifolds, Amer. Math. Soc. Colloq. Publ., Vol. 32, Providence, I. D., 1949.

Institute of Science and Technology, The University of Michigan and The University OF Michigan 